\title{
Neurocognitive mechanisms modulating attention bias in anxiety: Current
}

perspectives.

Guest editors: Hadas Okon-Singer ${ }^{1}$ and Tatjana Aue ${ }^{2}$

${ }^{1}$ University of Haifa, Israel

${ }^{2}$ University of Bern, Switzerland

\section{${ }^{*}$ Correspondence:}

Hadas Okon-Singer

University of Haifa

Department of Psychology

Mount Carmel

Haifa 3498838

Israel

hadasos@psy.haifa.ac.il

Phone: +972 48249671 


\section{(Editorial)}

\section{Title: Neurocognitive mechanisms modulating attention bias in anxiety: Current perspectives.}

Many studies have explored the mutual influences of attention and anxiety. The current special issue adds thirteen contributions from leading research groups worldwide, describing the role of different attention systems in children, adolescents and adults, in different types of anxious states and traits. These papers use a wide array of different methods, including fMRI, ERP, autonomic response and eye-tracking measurement, as well as innovative designs, in order to allow insights about psychophysiological mechanisms. The current special issue is linked to a second special issue (link) reporting work on cognitive biases other than attention biases, as well as the link among them in anxiety. This continues current attempts to describe less-studied biases and explore their causal linkages (Aue \& Okon-Singer, 2016). Taken together, insights from these two special issues suggest new and exciting directions for further research and treatment

There is evidence showing that different factors, including attention and personality traits and states, modulate emotional reactions (Okon-Singer, Lichtenstein-Vidne, \& Cohen, 2013; Rohr et al., 2016). This modulation may depend, however, on task-settings as illustrated by Lichtenstein-Vidne et al. (2016) who used a task that differentiates between task-relevant and task-irrelevant features of distracting items. Patients with anxiety or depression, and matched controls, were asked to perform a central task and ignore distracting pictures. While all participants showed a congruency effect to the task-relevant non-emotional aspect of the distractors, only anxious patients showed a bias towards negative distracting pictures, suggesting low attentional control among anxious patients. Corroborating these findings, Dodd et al (2016) used an elegant manipulation of task-relevance in a modified visual search task. The task had two versions: In the emotion-relevant task, participants responded according to the emotional expression of a target face. In the emotion-irrelevant version, participants were asked to respond according to the age of the same discrepant face and ignore its facial 
expression. Trait anxiety modulated visual search performance only in the emotion-irrelevant task, where a positive correlation was found between trait anxiety and a bias for angry faces.

Several studies in this issue focused on the mechanisms underlying attention biases in anxiety. The findings of Rossi et al. (2015) suggest that attention bias in anxiety is evident at the earliest processing stage already. Using ERPs, the authors showed that the amplitude of $\mathrm{C} 1$ - the first component of the visual evoked potential - is modulated by state anxiety and task demands: Anxiety increased the C1 to irrelevant stimuli and reduced the P300 to target stimuli. The work of Schroder et al. (2016) further shows abnormalities in early processing: Anxiety was related to abnormal brain activity prior to an erroneous response, as reflected in the errorpreceding positivity (EPP) component. Attenuated EPP amplitude among female participants with high worry levels suggests reduced pre-error disengagement of task-focused attention. Together, the results of these studies suggest that attention bias in anxiety is robust, impacts early processing, and deteriorates goal-directed performance.

Another study exploring the mechanisms at the basis of anxiety is that of Kret et al. (2016), who assessed attention bias in social anxiety using eye tracking. High socially-anxious individuals attended more to hand regions and less to the head region compared to low socially-anxious individuals. Future work may explore whether this pattern is limited to social anxiety, or whether a similar bias exists in other types of anxiety. van Dillen et al. (2016) used eye-tracking to examine the relation between social anxiety, moral integrity threats and affirmations, and avoidance of social threat. Participants were asked to retrieve an immoral, moral or neutral past act. Then, they passively viewed angry, happy and neutral faces. Selective avoidance of angry eyes was found following retrieval of an immoral memory, and was correlated with social anxiety. Retrieval of a moral memory eliminated this bias, regardless of social anxiety level. These findings underscore the impact of social context when examining emotional/cognitive biases.

Most of the evidence about the relationship between attention and anxiety is correlational. Three studies in the current issue examined the causal impact of attentional 
mechanisms on anxiety symptoms. In an innovative design, Birk and colleagues (2016) examined the impact of attentional control - reflected in the ability to inhibit distractors in a visual search task - on anxiety levels. The authors induced anxiety and measured autonomic arousal (measured by skin conductance), subjective anxiety and attentional control pre and post the anxiety induction. Low attentional control, seen as higher pre-induction distractibility in the visual search task, predicted higher subsequent autonomic arousal, suggesting a causal influence of attentional control on anxiety. Attentional control was suggested to have a causal role in models of cognitive biases in depression (Everaert et al., 2016). The findings of Birk et al. propose a similar mechanism in anxiety. Notably, since the findings of Lichtenstein-Vidne and colleagues (2016) show that patients with anxiety and depression differ in their attentional control, the underlying mechanisms may be modulated by task requirements. Two papers in this special topic employed an attention bias modification technique (ABMT) to examine the causal role of attention biases in anxiety. Interestingly, most previous studies that showed an impact of ABMT on anxiety symptoms trained individuals to shift attention away from negative stimuli. In contrast, these two studies used a different approach: Sass et al. (2016) examined whether enhancing attention bias towards positive stimuli optimizes training outcomes. Participants with high worry or physiological arousal levels showed reduced worry symptoms following training, associated with changes in both early and later attention-related mechanisms as reflected in ERPs (P100 and P300). These results complement those of Pintzinger et al. (2016) who measured ERPs in healthy individuals performing a dot-probe task. All participants showed attentional bias towards positive stimuli, as reflected in larger N1 amplitudes following positive vs. negative cues. Wald et al. (2016) showed that training combat soldiers to orient attention towards threatening stimuli immediately before combat reduced the association between combat exposure and stress-related symptoms at a two-month follow-up. These results highlight the role of attention bias in anxiety and the therapeutic potential of individually-tailored ABMT.

The study of Wald and colleagues (2016) emphasizes the importance of intervening prior to a negative life event. Therapeutic interventions may play a crucial role among anxious 
children and adolescents. Indeed, several papers in this special issue show such attention biases in vulnerable populations. Fu et al. (2016) used fMRI to explore the neural basis of attentional biases among children with behavioral inhibition (biologically-based hyper-vigilance to novelty). Using a dot-probe task, they found a relation between behavioral inhibition, anxiety, and activation in the dorsolateral prefrontal cortex. The authors concluded that these findings suggest that the link between prefrontal activation and anxiety might be related to earlydeveloping temperament vulnerabilities. Cohen et al. (2016) recently showed that attentional control training resulted in increased prefrontal-amygdala connectivity. These findings propose that attention training may be beneficial to anxious and behaviorally-inhibited individuals. They further underscore the need for future investigations in order to better understand the impact of training different attention sub-systems (e.g., orienting, arousal, executive control), using different protocols (bias towards or away from positive or negative stimuli, prior or after negative events). Troller-Renfree et al (2016) examined attentional biases in a dot-probe task among children from institutions who were randomized at an early age to receive either a highquality foster care or care-as-usual. At age 12, stability of foster care placement was related to positivity bias, while instability of foster care placement was related to threat bias. The magnitude of the positivity bias was further related to fewer internalizing problems and better coping mechanisms. Finally, Shechner et al. (2015) examined the relationship between anxiety, age, and attentional biases in two tasks: In one task, healthy and anxious youth and adults viewed a central picture and were asked to ignore distracting threatening or rewarding stimuli, while their fixations were tracked. In a second task, pupil diameter change was recorded following presentation of negative and neutral words. Using this approach, Shechner et al. (2015) show an impact of both age and anxiety on attentional biases.

In sum, the findings summarized here highlight new insights that have implications for future research and clinical applications. Attentional biases in anxiety are robust, evident already early processing stages, and happen even when irrelevant to the task-settings. These biases may result - at least in some cases - from focusing on different cues compared with healthy individuals and are related to abnormal prefrontal activation. Finally, evidence for a 
causal impact of attentional mechanisms, specifically attentional control, on anxiety symptoms, suggests targets for future therapeutic interventions, such as individually-tailored ABMT.

\section{Acknowledgements}

We would like to show our appreciation to all contributors for their enthusiastic commitment throughout the whole publication process of this special issue. We are also indebted to the implicated reviewers who, by giving numerous invaluable pieces of feedback, made the special issue substantially increase in quality. Finally, we are grateful to the Editorial Board and Staff of Biological Psychology and Elsevier Publications, in particular to the Editorin-Chief Ottmar Lipp, who encouraged us to realize the idea of this special issue. 


\section{References}

Aue, T., \& Okon-Singer, H. (2015). Expectancy biases in fear and anxiety and their link to biases in attention. Clinical psychology review, 42, 83-95.

Birk, J. L., Opitz, P. C., \& Urry, H. L. (2015). Distractibility as a precursor to anxiety: Preexisting attentional control deficits predict subsequent autonomic arousal during anxiety. Biological psychology.

Cohen, N., Margulies, D. S., Ashkenazi, S., Schäfer, A., Taubert, M., Henik, A., ... \& OkonSinger, H. (2016). Using executive control training to suppress amygdala reactivity to aversive information. Neurolmage, 125, 1022-1031.

Dodd, H. F., Vogt, J., Turkileri, N., \& Notebaert, L. (2016). Task relevance of emotional information affects anxiety-linked attention bias in visual search. Biological psychology.

Everaert, J., Grahek, I., \& Koster, E. H. (2016). Individual differences in cognitive control over emotional material modulate cognitive biases linked to depressive symptoms. Cognition and Emotion, 1-11.

Fu, X., Taber-Thomas, B. C., \& Pérez-Edgar, K. (2015). Frontolimbic functioning during threatrelated attention: relations to early behavioral inhibition and anxiety in children. Biological psychology.

Kret, M. E., Stekelenburg, J. J., de Gelder, B., \& Roelofs, K. (2015). From face to hand: Attentional bias towards expressive hands in social anxiety. Biological psychology.

Lichtenstein-Vidne, L., Okon-Singer, H., Cohen, N., Todder, D., Aue, T., Nemets, B., \& Henik, A. (2016). Attentional bias in clinical depression and anxiety: the impact of emotional and non-emotional distracting information. Biological psychology.

Okon-Singer, H., Lichtenstein-Vidne, L., \& Cohen, N. (2013). Dynamic modulation of emotional processing. Biological psychology, 92(3), 480-491. 
Pintzinger, N. M., Pfabigan, D. M., Pfau, L., Kryspin-Exner, I., \& Lamm, C. (2016). Temperament differentially influences early information processing in men and women: Preliminary electrophysiological evidence of attentional biases in healthy individuals. Biological Psychology.

Rohr, C. S., Dreyer, F. R., Aderka, I. M., Margulies, D. S., Frisch, S., Villringer, A., \& OkonSinger, H. (2015). Individual differences in common factors of emotional traits and executive functions predict functional connectivity of the amygdala. Neuroimage, 120, 154-163.

Rossi, V., \& Pourtois, G. (2015). Someone's lurking in the dark: The role of state anxiety on attention deployment to threat-related stimuli. Biological psychology.

Sass, S. M., Evans, T. C., Xiong, K., Mirghassemi, F., \& Tran, H. (2016). Attention training to pleasant stimuli in anxiety. Biological psychology.

Schroder, H. S., Glazer, J. E., Bennett, K. P., Moran, T. P., \& Moser, J. S. (2016). Suppression of error-preceding brain activity explains exaggerated error monitoring in females with worry. Biological psychology.

Shechner, T., Jarcho, J. M., Wong, S., Leibenluft, E., Pine, D. S., \& Nelson, E. E. (2015). Threats, rewards, and attention deployment in anxious youth and adults: An eye tracking study. Biological psychology.

Troller-Renfree, S., McLaughlin, K. A., Sheridan, M. A., Nelson, C. A., Zeanah, C. H., \& Fox, N. A. (2016). The beneficial effects of a positive attention bias amongst children with a history of psychosocial deprivation. Biological psychology.

Van Dillen, L. F., Enter, D., Peters, L. P., van Dijk, W. W., \& Rotteveel, M. (2016). Moral fixations: The role of moral integrity and social anxiety in the selective avoidance of social threat. Biological psychology. 
Wald, I., Bitton, S., Levi, O., Zusmanovich, S., Fruchter, E., Ginat, K., ... \& Bar-Haim, Y. (2016). Acute delivery of attention bias modification training (ABMT) moderates the association between combat exposure and posttraumatic symptoms: A feasibility study. Biological psychology. 